
\title{
Research S Surate \\ Key Barriers to the Provision and Utilization of Mental Health Services in low-and Middle-Income Countries: A Scope Study
}

\section{Yaser Sarikhani}

Shiraz University of Medical Sciences

Peivand Bastani ( $\sim$ bastanip@sums.ac.ir)

shiraz university of medical sciences https://orcid.org/0000-0002-0412-0267

\section{Mohammad Rafiee}

Shiraz University of Medical Sciences

\section{Zahra Kavosi}

Shiraz University of Medical Sciences

\section{Ramin Ravangard}

Shiraz University of Medical Sciences

\section{Research}

Keywords: Mental Health, Mental Health Services, Access, Utilization, Provision, Barriers

Posted Date: December 17th, 2019

DOl: https://doi.org/10.21203/rs.2.19025/v1

License: (c) (i) This work is licensed under a Creative Commons Attribution 4.0 International License. Read Full License

Version of Record: A version of this preprint was published at Community Mental Health Journal on April 13th, 2020. See the published version at https://doi.org/10.1007/s10597-020-00619-2. 


\section{Abstract}

Background: Mental Health( $\mathrm{MH})$ is among the main determinants of community well-being and a remarkable portion of global burden of diseases is pertaining to mental disorders. Despite this fact, little attention has been given to the provision of adequate and appropriate $\mathrm{MH}$ services and improving equitable access to these services in some parts of the world especially in low-and middle-income countries(LMICs). This review was aimed to identify key barriers to the provision and utilization of $\mathrm{MH}$ services in LMICs.

Methods: We carried out a systematic and comprehensive search on 7 important online databases for English-language literature on key barriers to the provision and utilization of MH services in LMICs from Jan 2000 to Nov 2019. Arksey and O'Malley instructor was used in 5 steps to implement scope study. The extracted materials were tabulated and synthesized using a qualitative content analysis approach.

Results: Three main themes were developed regarding the barriers to the provision of $\mathrm{MH}$ services in LMICs including resource and administrative barriers, information and knowledge obstacles, as well as policy and legislation barriers. Also four main themes emerged about barriers to the utilization of $\mathrm{MH}$ services including attitudinal, structural, knowledge, and treatment-related barriers.

Conclusion: Equitable access to MH services in LMICs is under influence of many barriers in both provision and utilization sides. In order to alleviate these problems, health systems in LMICs could adopt some strategies including integration of $\mathrm{MH}$ policy into general health policy, improvement of public $\mathrm{MH}$ knowledge regarding the $\mathrm{MH}$ and its related issues, developing anti-stigma programs, reallocation of health resources toward high-priority $\mathrm{MH}$ needs, developing community-based insurance, as well as integration of $\mathrm{MH}$ services into all levels of health-care systems. Further studies are also required for determining relative importance of the barriers and their related solutions based on the national context of each country.

\section{Introduction}

Mental health $(\mathrm{MH})$ is among the leading determinants of people's overall well-being (1). Mental disorders are among the main non-fatal causes of burden of diseases in the world (2), so that the burden of mental illnesses has become a great public health concern (3). Studies on global burden of disease show that more than 5 percent of Disability Adjusted Life Years (DALYs) and up to 15.7 percent of Years Lived with Disability (YLDs) in the world are associated with the mental disorders (4). Also economics-related health studies estimated that the global cost of mental disorders in 2010 was equal to $\$ 2.5$ trillion, and it was predicted that in 2020 the cost will increase to more than $\$ 6$ trillion (5).

Despite the increasing trend of global burden of mental illnesses, the problem has not been regarded appropriately like physical diseases and to a large extent has been neglected in many parts of the world specially in low-and middle-income countries (LMICs) (6-8). This defect leads to limited, inadequate, and inequitable provision of $\mathrm{MH}$ services (6), and would expand treatment gap. This widening gap causes 
many health, social, and economic complications (9). Studies have shown that key barriers to the provision of $\mathrm{MH}$ services include problems related to information system $(10,11)$, economic and resource barriers $(12,13)$, defects in evidence-based policy and practice (14), as well as prevention, integration, and structural barriers (14-16).

In addition to the provision of $\mathrm{MH}$ services, utilization of these services has a significant effect on $\mathrm{MH}$ condition of the community. Utilization of MH services is influenced by several factors (17). Studies have identified several barriers to the utilization of MH services, such as fear of stigma (18-20), transportation problems $(21-23)$, cost of services $(21,24,25)$, and information barriers $(13,17,18)$.

Since equitable access to health care services is affected by both supply and demand sides (26), in order to provide a pervasive view of the issue, identifying barriers to the utilization and provision of $\mathrm{MH}$ services is essential for evidence-based planning, priority setting for resource allocation, and finally reducing burden of mental disorders (21). Despite the fact that the majority of global burden of MH diseases is in LMICs (27), less than $10 \%$ of published researches on MH are form these countries (28). Also, based on our searches, there is not any comprehensive review in which barriers on the both supply and demand sides have been investigated simultaneously in LMICs. Therefore, this review scopes studies on barriers to the utilization and provision of MH services in LMICs in order to summarize, categorize and then discuss about each of the barriers that have extracted from the reviewed reports.

\section{Methods}

The main objective of this scoping review was to map the literature about key barriers to the utilization and provision of mental health services in LMICs simultaneously. We have used scoping review because this method allows inclusion of literatures with heterogeneous designs and samples (29). Also This type of review provides the possibility of identifying main aspects and factors related to the concept, building on a comprehensive map of the evidences on the issue, and determining knowledge gaps in the area (30).

In this regards, we used scoping review approach developed by Arksey and O'Malley (29). This methodology is consisted of five distinctive stages: 1- Identifying research question, 2- Finding the relevant studies, 3-Selecting relevant studies, 4 - Charting the data, as well as 5 - Collating, summarizing and reporting the results.

\section{Identifying the question}

Although research question determines scope of the study, but scoping review has an iterative process. Therefore, the research question of the study is developed gradually through the literature review process. Outcome of interest in our study was key barriers to the utilization and provision of $\mathrm{MH}$ services recognized by providers and patients in LMICs. In this study, adult population was considered as care receivers and in the provider side, all levels from individual caregivers to policy sectors were included. Therefore, this study aimed to answer to this question: 'what are the key barriers to the utilization and provision of MH services in LMICs?' 


\section{Finding the relevant studies}

Before carrying out a comprehensive review, we searched Cochrane database to ensure that there is no similar review. In order to find relevant studies, we conducted a systematic search on 7 online databases including PubMed, Scopus, Web of Science, Embase, ProQuest, Wiley online library, and Sciencedirect. We searched these databases for the relevant studies published from January 2000 to November 2019. We determined three categories of search term through an initial literature review and then we refined and completed categories during the systematic search process. Search terms in each category were combined using logical operator 'OR' and the categories were merged applying logical operator 'AND'. The search strategy of the study is shown in table 1. Using this search strategy, we have retrieved 71832 reports. After removing duplicates, 50131 papers were entered into the assessment phase. We used EndNote manager software (EndNote X7.1 version, by Thomson Reuters) to manage references.

\section{Selecting relevant studies}

For selecting the studies that were relevant to the research question, we carried out an iterative three-step peer review process, so that in each phase we refined the search strategy, searched literature, and reviewed new papers. For developing the key objective and question of the review and also in all steps of appraisal and inclusion we used three main criteria of scoping review studies. In this regard, adult patients, barriers to the provision and utilization of MH services, as well as LMICs were considered as "Population, Concept, and Context" (PCC) respectively. At the first step, title of papers was screened by two reviewers independently based on outcome of interest. In this step, 11237 studies were accepted for further assessment. After excluding irrelevant titles, abstract of remained papers were reviewed by two reviewers and those that did not meet the aim of the study were removed and 163 full-text articles were selected for further appraisal. Finally, 2 reviewers scanned full-text papers and 35 studies including 20 articles on barriers to the provision of MH services $(10-13,15,20,23,31-43)$ and 24 studies on challenges of utilization of MH services $(13,17,18,20,23-25,31,33,35,37,39,42,44-54)$ were selected for final analysis. "Critical Appraisal Skills Programme" (CASP) checklists (55) and "Strengthening the Reporting of Observational Studies in Epidemiology" (STROBE) tools (56) were used for quality assessment of selected full-texts. In all steps of selection phase, cases of disagreement were reviewed by a third reviewer for final inclusion. In order to get more familiar with all steps of the research process, two reviewers involved in a pilot project prior to the implementation of main study.

\section{Inclusion/exclusion criteria}

Due to the language limitations, only studies with at least an abstract in English were included. Since healthcare systems have changed remarkably over the recent years, and in order to investigate the latest barriers to the provision and utilization of MH services in LMICs, articles published after 2000 were included. We included original researches and discussion papers, however commentaries, letters, and reviews of other studies were excluded.

Figure 1 shows the process of paper selection for this study in the form of a PRISMA flowchart. 
Figure 1-PRISMA Flowchart of the included papers in the scope study

\section{Charting the data}

We applied a content analysis approach for charting the data (57). We developed a data-charting form to extract relevant data from included papers. In this regards two reviewers carried out the charting jointly through an iterative process, so that they extracted data and updated the data-charting form continuously.

\section{Collating and summarizing the data}

We analyzed extracted data using a qualitative thematic analysis (58). At the first step of thematic analysis, we became familiar with the data through reading and re-reading the full-text papers. Then we identified preliminary codes based on research question and outcome of interest. In the third step, we conducted an interpretive analysis of the initial codes and organized them into sub-themes and main themes. Reviewing themes was the next step. In this regards, we carried out a deeper review on the identified themes in order to combine, refine, separate, or discard initial themes if it was necessary. In the final step, we defined and labeled themes and their related sub-themes in terms of relevancy of the contents.

The main themes and their related sub-themes are presented in the format of tables. Also, in order to provide a better view of the breadth of evidence, frequency of studies involved in development of each theme and sub-theme are listed in the tables. Finally, we generated a thematic map of the evidence in order to provide a more comprehensive insight into the barriers to equitable access to $\mathrm{MH}$ services.

\section{Results}

Our searches resulted in the retrieval of 71832 publications, of which 35 articles were finally included in the review. Table 2 shows the characteristics of included studies. A large portion of included studies were from Africa and the majority of articles performed using a qualitative or mixed-method design. Most of the studies on utilization (70\%) and provision (50\%) conducted with the participation of healthcare professionals. Thematic analysis resulted in development of 3 main themes and 13 sub-themes regarding the barriers to the provision of $\mathrm{MH}$ services which are presented in table 3 . Also, results of thematic analysis lead to 4 main themes and 20 sub-themes regarding the barriers to the utilization of $\mathrm{MH}$ services which are presented in table 4.

\section{A. Barriers to the provision of $\mathrm{MH}$ services}

\section{A-1-Resource and administrative barriers}

A-1-1- Insufficient resources: results of the analysis showed that lack of sufficient resources was the most frequent barrier regarding the provision of $\mathrm{MH}$ services. Findings indicated that scarcity of resources for $\mathrm{MH}$ services in LMICs can be classified into four main categories including restricted financial resources 
$(12,15,33,35,39)$, shortage of adequate MH professionals (33-35, 38-40), insufficiency of medications (42), and limited healthcare facilities $(13,33,42,43)$.

A-1-2- Geographical imbalance in resource allocation: imbalanced geographical distribution of $\mathrm{MH}$ services was another frequent barrier to the provision of $\mathrm{MH}$ services. Studies investigated this problem indicated that inequality in distribution of $\mathrm{MH}$ resources is pertaining to the unequal distribution of budgetary resources $(12,13)$, centralized $\mathrm{MH}$ beds and facilities $(10,12,41,43)$, and unbalanced distribution of MH staff $(11,12)$.

A-1-3- Centralized and non-integrated services: this review showed that one of the main barriers to equitable provision of MH services in many LMICs is that these services are usually not integrated into Primary Health Care (PHC) and a major proportion of service facilities are located in the centers of provinces $(10,12,13,20,31,38,40)$.

A-1-4- Inappropriate service types: findings of current study revealed that failure to provision of appropriate MH services is a challenges for health systems in some LMICs. Inappropriateness of $\mathrm{MH}$ services is related to the improper allocation of $\mathrm{MH}$ services resources $(13,40,52)$ as well as incongruity between available services and needs or preferences of target population $(15,43)$.

A-1-5- Imperfect inter-organizational collaboration: lack of appropriate cooperation between different parts of the health system associated with $\mathrm{MH}$ services was another challenges for provision of $\mathrm{MH}$ care in developing countries. Studies reported that this problem has different aspects including lack of cooperation between scientific institutions for development of preventive and treatment programs (20), poor coordination between policy and practice (11), and lack of cooperation between organizations and professionals at different levels of $\mathrm{MH}$ services $(10,11)$.

A-1-6- Weakness of quality assurance Programs: this review showed that another challenge of health systems in LMICs for provision of MH services is deficiency of quality assurance programs. Quality assurance practices usually focus on professionals' competency, quality of equipment and medications, and provision of evidence-based services $(11,35)$.

\section{A-2-Information and knowledge barriers}

A-2-1- Inappropriate professionals' training: findings of the study indicated that inappropriate or inadequate training of professionals in LMICs is the most frequent knowledge barrier to the provision of high-quality MH services. This problem can be arisen in all professionals from different levels and regarding all necessary skills such as diagnosis and treatment $(21,23,32,38,40,42)$.

A-2-2-Imperfect mental health information system: analysis showed that defective $\mathrm{MH}$ information system is a barrier to the provision of appropriate and equitable $\mathrm{MH}$ services in many developing countries. This deficiency has an influence almost on all aspects of provision of $\mathrm{MH}$ service $(11,13,20$, $36,39)$. 
A-2-3- Weakness of evidence-based practice: findings of this study indicated that weakness in evidencebased $\mathrm{MH}$ practice is another knowledge barrier to the provision of $\mathrm{MH}$ services. Problem of dissemination of researches evidence and lack of appropriate national guidelines are among the main aspects of the barrier in LMICs $(10,12,13,38,43)$.

\section{A-3- Policy and legislation barriers}

A-3-1- Low priority of mental health in health policy: this review revealed that low priority of $\mathrm{MH}$ in national health policy is the most frequent policy barrier to the provision of equitable $\mathrm{MH}$ services in some LMICs. Limited financial, physical, and human capacities, imperfect information system, as well as restricted participation of stakeholders such as patients and their advocates are highly associated with the problem $(10-13,32,34,39)$.

A-3-2- Weakness of evidence-based policy making: this study showed that deficient MH information system, restricted infrastructures, as well as limited capacities resulted in weakness of evidence-based policy in many LMICs. Policy weakness has a direct association with decreased quantity and quality of $\mathrm{MH}$ services in this countries $(11,13,32,34)$.

A-3-3- Imperfect legislation on insurance: we found that one the barriers to the provision of $\mathrm{MH}$ services in LMICs is poor legislation on insurance. Providing equitable insurance coverage for mental diseases, as a critical duty of health systems, needs precise legislation in many developing countries $(15,20)$.

A-3-4- Inappropriate legislation on mental health services: according to the findings of this study inappropriate legislation and law enforcement regarding the provision of $\mathrm{MH}$ services was a challenge for health systems in LMICs. Accurate legislation for $\mathrm{MH}$ is considered as an important stewardship function of health systems $(34,43)$.

\section{B. Barriers to the utilization of $\mathrm{MH}$ services}

\section{B-1-Attitudinal barriers}

B-1-1- Concern about social stigma: based on the findings of this review, the main attitudinal obstacle to the utilization of $\mathrm{MH}$ services among patients is the concern about being stigmatized by others. A large body of included studies (85\%) has reported that fear of being stigmatized hindered people from seeking MH services $(17,18,20,23-25,31,33,35,37,39,42,44,45,48-50,52-54)$.

B-1-2- Personal stigma/Shame: according to the findings of current review, self-stigma and embarrassment is the second attitudinal barrier to the utilization of $\mathrm{MH}$ services. Self-stigma is a feeling of shame that in many cases leads to reduction in utilization of MH services $(18,20,44,48,50,52-54)$.

B-1-3- Concerns about the effectiveness of services: this study indicates that another frequent barrier to the utilization of MH services in LMICs is concern about the effectiveness of services. Patients who 
suppose that MH service would not help them are less likely to seek and utilize these services $(18,23-25$, $48,49)$.

B-1-4- Concerns about attitude and behavior of professionals: results of this review showed that negative behaviors and attitudes of $\mathrm{MH}$ care providers toward patients is an important underlying factor that affect utilization of available $\mathrm{MH}$ services. This problem is pertaining to the past negative experiences with $\mathrm{MH}$ professionals $(17,18,20,23,42,54)$.

B-1-5- Willingness to take alternative forms of care: the results demonstrated that patients' preferences for alternative types of treatment is an attitudinal barrier to the utilization of formal MH services. Seeking help from religious and traditional healers is a prevalent issue in many LMICs that prevents patients from utilization of existing MH services $(18,23,31,33,49)$.

B-1-6- Cultural beliefs against treatment: the findings indicated that in many developing countries mental illnesses are attributed to the spirit (35). Therefore, some cultural beliefs such as fatalism (52) create a gap between personal beliefs and $\mathrm{MH}$ interventions that eventually leads to reduced utilization of $\mathrm{MH}$ services $(23,35,48,52)$.

B-1-7- Patients' self-reliance: this study showed that because of previous negative experiences or due to the concerns about stigma, patients prefer to handle their problem on own or may decide to wait for the disease to get better by itself $(17,18,49)$.

B-1-8- Lack of confidence in professionals: results of the review demonstrate that due to the unsuccessful or bad experience with $\mathrm{MH}$ care providers, patients may lose their confidence in professionals and may reduce utilization of available services $(17,48,50)$.

B-1-9- Poor compliance with treatment: this study showed that the last attitudinal barrier to the utilization $\mathrm{MH}$ services is patients' poor compliance with treatment. Patients who think interventions are merely to control their behavior and are not effective for their treatment may have little adherence to the cares (37).

\section{B-2-Structural barriers}

B-2-1- Cost of services: this review indicated that the second most frequent (70.8\% of studies included) barrier to the utilization of MH services in LMICs was cost of services. Although in some LMICs suitable $\mathrm{MH}$ services are available, cost of these services is not affordable for patients and their families $(13,17$, $18,23-25,31,33,35,39,42,44-46,49,51,54,59)$.

B-2-2- Location, distance and transportation barriers: the findings revealed that challenges related to the transportation and location of existing $\mathrm{MH}$ services were the second structural barrier to the utilization of services in many LMICs. This problem is more dominant in the remote and real areas of these countries $(13,17,18,23,25,44,47,49,54)$. 
B-2-3- Lack of family or social support: we found that shortage of support from others is a deterrent factor against utilization of $\mathrm{MH}$ services in some developing countries. Social support of patients with mental illness has different dimensions including financial, emotional, and physical supports $(18,33,35$, $45,48,49,52,53)$.

B-2-4- Time-related barriers: this study showed that challenges pertaining to time are another structural barrier to the utilization of MH services in LMICs. These challenges include waiting time barriers, difficulty taking time off work, and transportation time. $(17,18,37,42,44,49)$.

B-2-5- Inappropriate and inflexible services: this review indicated that some MH services in LMICs are not convenient enough for patients. Inappropriateness of services is attributable to inconvenient hours of service provision, inflexible appointments, fragmented service delivery system, as well as undesirable medications and interventions $(17,18,42,45,49)$.

B-2-6- Lack of adequate services: findings of this study demonstrated that due to the shortage of financial, human, and physical resources, provision of equitable and well-distributed $\mathrm{MH}$ services is a great problem in many LMICs. Accordingly, lack of adequate MH services has a definite negative effect on access to the services $(20,23,25,52,54)$.

B-2-7- Limited insurance coverage: the results indicated that poor health insurance system in LMICs is a deterrent factor against equitable access to $\mathrm{MH}$ services. Imperfect insurance legislation, incomplete basic premium package, low population coverage, and unaffordable premiums are among the most frequent deficits of $\mathrm{MH}$ insurance in these countries $(20,23,37,39,42)$.

\section{B-3-Knowledge barriers}

B-3-1- Lack of knowledge about mental health problem: this study showed that the most frequent knowledge barrier to the utilization of MH services in LMICs is lack of adequate knowledge about $\mathrm{MH}$ problems among patients and their families. Recognition of $\mathrm{MH}$ problems by patients and their families is the first step in utilization of the services $(17,18,25,35,42,45,48-52)$.

B-3-2- Lack of information on available services: This study indicated that lack of information about availability of services is another important knowledge barrier to the utilization of MH services. This problem is pertaining to the lack of information about various types of treatment and place of services $(17,18,25,44,49)$.

\section{B-4- Treatment-related barriers}

B-4-1- Fear of treatment side effects: results of the study showed that patients' concern about side effects of available treatments has negative impact on utilization of $\mathrm{MH}$ services. Treatment side effects are mainly related to medications and technology-based interventions $(18,23,45,46,48,49)$. 
B-4-2- Long-term nature of treatment: findings of the study indicated that long-term period of $\mathrm{MH}$ treatments is another barrier to the utilization of $\mathrm{MH}$ services. Long-dated hospitalizations, repeated outpatient consultations, and delayed treatment outcomes may induce patients to give up treatments (45).

All the relationships between the main themes and subthemes are clarified in the thematic network (Figure 2).

\section{Figure 2- The thematic network of the Key Barriers to the Provision and Utilization of Mental Health Services}

\section{Discussion}

This review was carried out to determine barriers to the provision and utilization of MH services in LMICs. Since equitable access to $\mathrm{MH}$ services is under influence of barriers at both supply and demand sides, we investigated challenges regarding the utilization and provision of $\mathrm{MH}$ services simultaneously in order to provide a more comprehensive perspective on the issue. In this section, we discussed the findings of the study based on each theme and its related sub-themes. Due to the publishing limitations, we focused especially on the most frequent barriers.

This review showed that scarcity of resources for $\mathrm{MH}$ services is a major problem for health systems in many LMICs. Limitation of resources in $\mathrm{MH}$ is the result of poor economic condition, low priority of $\mathrm{MH}$, and deficient stewardship (10). Insufficiency of resources for providing MH services can be categorized as inadequate financial resources $(12,39)$, lack of enough professionals $(40)$ particularly at the primary level (60), shortage of medications (42), and limited service delivery facilities (43). Countries that are faced with shortage of financial resources in $\mathrm{MH}$ have to reallocate resources in order to meet their high priority needs in mental health (14). Evidence-based resource allocation in LMICs should be done considering the cost-effectiveness of $\mathrm{MH}$ services(12,41). Furthermore, efficiency and effectiveness of existing financial resources should be assured for provision of sustainable MH services (61).

Human resources are the core component for provision of $\mathrm{MH}$ services. Insufficiency of human resources may occur at both primary and special levels (60). At the primary level, delivery of MH services is focused mainly on non-specialist workers (14). Generally, Shortage of MH staff at this level is due to the limited number of educated workforces and unbalanced distribution of existing resources inside the country (62). At the specialty level most challenges regarding the shortage of $\mathrm{MH}$ workforces are related to rural-tourban movement (60) and migration to the countries with possible higher income (63). An appropriate planning and policy on MH human resources is necessary in LMICs for training adequate and qualified workforces, retaining them, and distributing $\mathrm{MH}$ care professionals equitably (60). Shortage of $\mathrm{MH}$ medications is considered as a great challenge in some developing countries (32). Countries that are facing this problem have to improve their pharmaceutical supply chain in order to provide low-cost drugs with the highest possible quality $(11,13)$. Moreover, in countries with severe limitation of resources, redistribution of financial resources is necessary for procurement of essential MH medications (11). 
Result of this study indicated that concentration of $\mathrm{MH}$ care facilities in urban areas and non-integrated $\mathrm{MH}$ services are among the main challenges of providing equitable MH cares in many LMICs. The main challenges of integrating $\mathrm{MH}$ services into PHC are lack of adequate infrastructures, insufficient $\mathrm{MH}$ human resources, and absence of appropriate initiatives in this regard $(64,65)$. Implementation of multidimensional interventions are necessary for integrating $\mathrm{MH}$ services into $\mathrm{PHC}$ and decentralizing the services (64). Health systems should adopt appropriate policies for re-distribution of $\mathrm{MH}$ resources toward community-based services such as outpatient clinics and community oriented inpatients facilities $(13,31,41)$. Despite the great emphasis on integrating mental health services in to PHC, there are also some issues that health systems need to be careful about them (10). The most important challenges in this regard are lack of adequate and well-trained professionals $(12,38,40)$, imposing overburden on staff at primary care level, failure in efficient supervision (12), lack of sufficient financial and physical resources, and deficient patient flow and referral processes $(12,40)$. Therefore, developing a practical and supportive referral system is necessary for integration of $\mathrm{MH}$ services into PHC (12).

Our review showed that provision of appropriate $\mathrm{MH}$ services is a concern for health systems in developing countries. In some cases, existing services are not compatible with the community needs (15). Appropriateness of the provided services should be considered from different perspectives such as type of services (preventive or therapeutic) (16), setting of services (inpatient or outpatient) (13), target population (Adults or children) $(43,66)$, equipment and medications $(10,40)$, as well as location and time of service delivery $(10,15)$. Health systems should reallocate $\mathrm{MH}$ resources in accordance with the community needs (10). Some suggestions in this regard include using appropriate technologies and medications based on health technology assessment analysis, improving community-based and integrated services, revision of existing services based on patient's preferences and convenience.

Professionals' competency in relation to the quality of training is a major knowledge barrier to the provision of MH services in the LMICs. Training may be inadequate among professionals at both primary and specialty levels $(23,32,42)$. Also, training may be insufficient regarding various skills necessary for patients' care including, prevention, diagnosis, and treatment (42). Another aspect of this problem is lack of sufficient knowledge on special groups like geriatric psychiatry (43). Inappropriate training leads to knowledge-practice gap and hinders provision of high quality services (40). Developing in-service training programs would be efficient in filling the gap $(38,40)$. Therefore, health systems in LMICs have to adopt a holistic approach to the patients' care when developing MH education (42).

Information system was introduced as a key building block of health care systems by World Health Organization (67). It is obvious that evidence-based MH policy, planning, education, and practice are dependent upon an efficient information system $(11,13,20,36)$. Furthermore, a well-developed $\mathrm{MH}$ information system has an important role in patients' registration and follow-up, monitoring health plans, and evaluating quality of services $(11,13,39)$. Shortage of logistic resources and lack of skillful staff are the basic challenges for establishment of $\mathrm{MH}$ information system in developing countries (68).

Governments have to increase investments in development and maintenance of $\mathrm{MH}$ information system. Involvement of all stakeholders such as policy makers, managers, professionals, and researchers is 
necessary for better development and adoption of the system $(36,68)$. A practical $\mathrm{MH}$ information system should have the capacity of combining and disseminating evidence in order to develop national guidelines for informed policy, practice and education $(13,38,43)$.

Rigorous $\mathrm{MH}$ policy is a neglected part of national health policy in some developing countries. Although high-income countries have had significant achievements in MH policy and practice (32), importance of $\mathrm{MH}$ as an integrated part of health policy has not yet been recognized properly in many poorer countries (14). This problem necessitates development of a comprehensive and evidence-informed MH policy in LMICs. Formulation of MH policy should be based on real needs of the community and governments should have adequate commitment to implementation of the policy in all levels (13). National MH policies must be formulated according to the condition of each country and it is necessary to develop compound indicators for assessment of policy at all levels from the adoption of policy to the implementation and practice (14).

This review indicated that, similar to the many other countries, the most important attitudinal barriers to the utilization of MH services in LMICs is fear of social stigma. One useful approach for detracting stigmatization is improving public knowledge about mental disorders (59). Also, it is suggested that interpersonal face to face communication between community individuals and people with mental disorders could be helpful (69). However, these strategies should be used according to the specific intermediaries of each situation (70). Moreover, it is suggested that developing some anti-stigma campaigns would be effective (19).

Self-stigma as another important attitudinal barrier to the utilization of $\mathrm{MH}$ services is related to the negative internalized perceptions and beliefs about MH status. This feeling can result in shame and accordingly reduces willingness to seek $\mathrm{MH}$ services (71). Forasmuch as, negative personal attitudes toward mental health problems is the leading cause of this problem, educating patients can alleviate it (20). Support from groups of peer who have controlled the problem successfully (72) and selfempowerment (73) are suggested as other approaches for tackling self-stigma.

Patients' concerns about usefulness and quality of services is another challenges of utilization of $\mathrm{MH}$ services in developing countries. Undesirable experience of treatment could affect patients to be worried about the effectiveness of services (21). Furthermore, patients' tendency towards alternative types of treatment may persuade them to become pessimistic about the specialized services $(18,31)$. According to these reasons, it seems that improving the quality of mental health services and increasing public knowledge about these services could remove or modify the barrier.

Concern about negative attitudes and behaviors of $\mathrm{MH}$ professionals is another attitudinal barrier to the utilization of MH services in LMICs. An unfavorable experience with care providers may have great impact on the patients' willingness to utilize available services $(17,54)$. Lack of adequate training $(18$, 20 ), failure to align scientific learning with cultural beliefs (23), and fear of working with patients with mental disorders are among the most important causes of professionals' negative attitudes and 
behaviors toward patients (42). Some researches indicated that implementation of stigma reduction programs for service providers would have positive outcomes $(18,54)$.

Unaffordable cost of $\mathrm{MH}$ services is the most frequent structural barriers to the utilization of available services in LMICs. The highest expenditures are pertaining to the medications and treatment services (74). A remarkable share of the cost is also attributable to the economic losses due to the reduced productive capacity of patients and their family (75). Some of the main reasons for this problem are lack of insurance coverage (21), imperfect coverage (76), and unaffordable insurance premium (33). In order to reduce direct costs of treatment, it is suggested that countries have to develop initiatives to introduce or improve community-based health insurance schemes (45). Providing affordable cost-effective interventions is another approach that would be helpful in alleviating the problem (77).

Location of $\mathrm{MH}$ care facilities and difficulties in transportation are among the most frequent structural barriers to the utilization of services in many developing countries. Geographical distance from $\mathrm{MH}$ facilities, especially in rural areas, has a notable adverse effect on equitable access to MH care in LMICs (78). In addition to the limited transportation capacity, cost of transportation as an indirect cost of treatment is also associated with limited access to $\mathrm{MH}$ services (75). It is suggested that integrating $\mathrm{MH}$ services into primary health care and developing more community-based facilities can reduce these barriers in developing countries (22).

In some LMICs lack of social support resulted in reduced access to MH care. Social support is a multidimensional issue. From one perspective, patients from poor families usually need financial support for utilization of available MH services (45). On the other hand, some patients need support for emotional relief which is known as "implicit support" (53). Finally, patients with severe illnesses need someone to help them get professional care $(18,49)$. In order to reduce these problems some strategies such as providing financial grant for poor families (23), improving peer support programs (53), as well as expanding adequate and community-based $\mathrm{MH}$ services (45) are suggested.

Time-related barriers are among the main structural challenges of access to $\mathrm{MH}$ services. Long waiting times at out-patient facilities and hospitals may persuade some patients to give up treatments(44). Another challenge is problem with taking time off work or home responsibilities for employees and housekeepers $(18,42,49)$. Transportation time is another time-related barrier that prevent access to $\mathrm{MH}$ services. Health systems in LMICs should give priority to $\mathrm{MH}$ and have to support $\mathrm{MH}$ services by providing adequate resources in order to promote treatment continuation (44).

Lack of adequate knowledge about mental problem among patients and their families is a great challenge against utilization of MH services in many developing countries. Recognition of the problem is an essential prerequisite for help-seeking and accordingly utilization of $\mathrm{MH}$ services (79). Patients' knowledge about the nature of their mental disorder, symptoms of the disease, and severity of the illness would lead to better recognition of the problem (80). Therefore, more severe cases of disorders are more likely to be identified and treated (81). Therefore, health systems in developing countries have to 
implement appropriate public education programs in order to improve public knowledge about $\mathrm{MH}$ disorders and their symptoms (18).

This study indicated that lack of information about different types of treatment and place of services are important knowledge barriers to the utilization of MH services in some LMICs. In line with our findings, results of a global MH survey conducted by WHO indicated that lack of knowledge about the existing services was a great barrier to the utilization of services (21). Health systems in developing countries should improve public knowledge about available MH services in order to help patients seeking treatment (44). Also, improving social support could be effective in raising awareness of patients and their families (18).

Past experience of treatment adverse effects, especially in medications and technology-based treatments, plays a deterrent role against utilization of $\mathrm{MH}$ services. Lack of adequate knowledge on necessity of continuous treatment and its possible adverse effects is a great challenge which dissuades patients from receiving care and decreases adherence to treatments $(45,46)$. It is suggested that increasing patients' knowledge about different treatment choices and their potential side effects would be beneficial (23).

\section{Research gaps}

This review indicated that some areas on barriers to the provision and utilization of MH services in LMICs are neglected and require further researches. One of these areas is difficulties in patient-provider relationship in $\mathrm{MH}$ services settings. Another field of research is problems of compliance with treatment among mental illness patients. In this review we discussed the results of analysis regarding the frequency of barriers. Therefore, relative importance of these barriers and their related solutions based on the national context of each country remains as an important area of further research.

\section{Study limitations}

In this review we included papers with various types of study and different design methods. This problem sometimes leads to difficulty in the synthesis of data. However, scoping review comprises an approach for appraisal and inclusion of heterogeneous studies. In this regard, a thematic content analysis can greatly alleviate the problem. Although we tried our best to review all studies pertaining to challenges of provision and utilization of MH services in LMICs, access to all relevant studies is dependent on various contributors with the potentiality of missing some evidences. Finally, in this review we focused on barriers to the provision and utilization of $\mathrm{MH}$ services among adult population. Therefore, this study did not consider other groups such as children or the elderly.

\section{Conclusion}

Equitable access to $\mathrm{MH}$ services is under influence of both supply and demand sides. On the supply side, provision of appropriate MH services in LMICs is subjected to some barriers including resource and administrative barriers, information and knowledge obstacles, as well as policy and legislation barriers. 
On the other side, patients in LMICs are faced with some barriers to the utilization of MH services including attitudinal, structural, knowledge, and treatment-related barriers. According to the these evidence, health systems in LMICs cloud adopt various strategies such as integration of MH policy into general health policy, reallocation of health resources in favor of high-priority $\mathrm{MH}$ services, developing community-based insurance with a special respect to $\mathrm{MH}$ services, integration of $\mathrm{MH}$ services into all levels of healthcare systems, development of comprehensive $\mathrm{MH}$ information system, improvement of public knowledge about $\mathrm{MH}$ and its related issues, and developing stigma reduction programs.

\section{Abbreviations}

$\mathrm{MH}$

Mental Health

LMICs

Low-and Middle-Income Countries

DALYs

Disability Adjusted Life Years

YLDs

Years Lived with Disability

$\mathrm{PHC}$

Primary Health Care

WHO

World Health Organization

\section{Declarations}

Ethics approval and consent to participate: The study`s proposal is approved by ethics committee affiliated with Shiraz University of Medical Sciences with the ID of SUMS-97-01-07-18586.

Consent for publication: Not applicable

Availability of data and material: All data in a form of data extraction sheets are available from the corresponding author on reasonable request.

Competing interests: All authors declare that they have no conflict of interest regarding this study.

Funding: This study was sponsored by Shiraz University of Medical Sciences under code (97-01-0718586). The funding body was not involved in the design of the study, data collection, analysis, and interpretation, as well as in writing the manuscript.

Authors' Contributions: PB, YS, ZK, and RR designed the study. YS, MR, and PB scanned the titles, reviewed the abstracts and appraised the full-texts papers. YS, PB, and MR wrote the present paper. PB, 
ZK, and RR critically revised the manuscript. All authors have read and approved the article for final submission.

Acknowledgements: Authors would like to thank Shiraz University of Medical Sciences for supporting to conduct the study.

\section{References}

1. WHO. The World Health Report 2001: Mental health: new understanding, new hope: World Health Organization; 2001.

2. Xia Y, Wu Q, Wang H, Zhang S, Jiang Y, Gong T, et al. Global, regional and national burden of gout, 1990-2017: a systematic analysis of the Global Burden of Disease Study. Rheumatology. 2019.

3. Mokdad AH. The burden of mental disorders in the Eastern Mediterranean region, 1990-2015: findings from the global burden of disease 2015 study. International journal of public health. 2017:113.

4. Kassebaum NJ, Arora M, Barber RM, Bhutta ZA, Brown J, Carter A, et al. Global, regional, and national disability-adjusted life-years (DALYS) for 315 diseases and injuries and healthy life expectancy (HALE), 1990-2015: a systematic analysis for the Global Burden of Disease Study 2015. The Lancet. 2016;388(10053):1603-58.

5. Bashshur RL, Shannon GW, Bashshur N, Yellowlees PM. The empirical evidence for telemedicine interventions in mental disorders. Telemedicine and e-Health. 2016;22(2):87-113.

6. Gilbert BJ, Patel V, Farmer PE, Lu C. Assessing development assistance for mental health in developing countries: 2007-2013. PLoS medicine. 2015;12(6):e1001834.

7. Sockalingam S, Arena A, Serhal E, Mohri L, Alloo J, Crawford A. Building provincial mental health capacity in primary care: An evaluation of a project ECHO Mental Health Program. Academic Psychiatry. 2017:1-7.

8. Pearson GS, Hines-Martin VP, Evans LK, York JA, Kane CF, Yearwood EL. Addressing gaps in mental health needs of diverse, at-risk, underserved, and disenfranchised populations: A call for nursing action. Archives of Psychiatric Nursing. 2015;29(1):14-8.

9. Rebello TJ, Marques A, Gureje O, Pike KM. Innovative strategies for closing the mental health treatment gap globally. Current opinion in psychiatry. 2014;27(4):308-14.

10. Knapp M, Funk M, Curran C, Prince M, Grigg M, McDaid D. Economic barriers to better mental health practice and policy. Health policy and planning. 2006;21(3):157-70.

11. Petersen I, Marais D, Abdulmalik J, Ahuja S, Alem A, Chisholm D, et al. Strengthening mental health system governance in six low-and middle-income countries in Africa and South Asia: challenges, needs and potential strategies. Health policy and planning. 2017;32(5):699-709.

12. Saraceno B, van Ommeren M, Batniji R, Cohen A, Gureje O, Mahoney J, et al. Barriers to improvement of mental health services in low-income and middle-income countries. The Lancet. 2007;370(9593):1164-74. 
13. Sulaberidze L, Green S, Chikovani I, Uchaneishvili M, Gotsadze G. Barriers to delivering mental health services in Georgia with an economic and financial focus: informing policy and acting on evidence. BMC health services research. 2018;18(1):108.

14. Jenkins R, Baingana F, Ahmad R, McDaid D, Atun R. International and national policy challenges in mental health. Mental Health in Family Medicine. 2011;8(2):101.

15. Martinez W, Galván J, Saavedra N, Berenzon S. Barriers to integrating mental health services in community-based primary care settings in Mexico City: a qualitative analysis. Psychiatric services. 2016;68(5):497-502.

16. Wainberg ML, Scorza P, Shultz JM, Helpman L, Mootz JJ, Johnson KA, et al. Challenges and opportunities in global mental health: a research-to-practice perspective. Current psychiatry reports. 2017;19(5):28.

17. Ghanizadeh A, Arkan N, Mohammadi M, Zarchi G, Ahmadi J. Frequency of and barriers to utilization of mental health services in an Iranian population. Eastern Mediterranean Health Journal 2008;14(2):438-46.

18. Luitel NP, Jordans MJ, Kohrt BA, Rathod SD, Komproe IH. Treatment gap and barriers for mental health care: A cross-sectional community survey in Nepal. PloS one. 2017;12(8):e0183223.

19. Salaheddin K, Mason B. Identifying barriers to mental health help-seeking among young adults in the UK: a cross-sectional survey. Br J Gen Pract. 2016;66(651):e686-e92.

20. Taghva A, Farsi Z, Javanmard Y, Atashi A, Hajebi A, Khademi M. Stigma barriers of mental health in Iran: A qualitative study by stakeholders of mental health. Iranian journal of psychiatry. 2017;12(3):163.

21. Andrade LH, Alonso J, Mneimneh Z, Wells J, Al-Hamzawi A, Borges G, et al. Barriers to mental health treatment: results from the WHO World Mental Health surveys. Psychological medicine. 2014;44(6):1303-17.

22. Brenes GA, Danhauer SC, Lyles MF, Hogan PE, Miller ME. Barriers to mental health treatment in rural older adults. The American Journal of Geriatric Psychiatry. 2015;23(11):1172-8.

23. Schierenbeck I, Johansson P, Andersson L, van Rooyen D. Barriers to accessing and receiving mental health care in Eastern Cape, South Africa. Health Hum Rights. 2013;15(2):110-23.

24. Borba CP, DePadilla L, McCarty FA, Silke A, Druss BG, Sterk CE. A qualitative study examining the perceived barriers and facilitators to medical healthcare services among women with a serious mental illness. Women's Health Issues. 2012;22(2):e217-e24.

25. Mwansisya TE, Outwater AH, Liu Z. Perceived barriers on utilization of mental health services among adults in Dodoma Municipality-Tanzania. Journal of Public Mental Health. 2015;14(2):79-93.

26. Richard L, Furler J, Densley K, Haggerty J, Russell G, Levesque J-F, et al. Equity of access to primary healthcare for vulnerable populations: the IMPACT international online survey of innovations. International journal for equity in health. 2016;15(1):64.

27. WHO. Atlas: child and adolescent mental health resources: global concerns, implications for the future: World Health Organization; 2005. 
28. Saxena S, Paraje G, Sharan P, Karam G, Sadana R. The 10/90 divide in mental health research: trends over a 10-year period. The British Journal of Psychiatry. 2006;188(1):81-2.

29. Arksey H, O'Malley L. Scoping studies: towards a methodological framework. International journal of social research methodology. 2005;8(1):19-32.

30. Munn Z, Peters MD, Stern C, Tufanaru C, McArthur A, Aromataris E. Systematic review or scoping review? Guidance for authors when choosing between a systematic or scoping review approach. BMC medical research methodology. 2018;18(1):143.

31. Ali SH, Agyapong VI. Barriers to mental health service utilisation in Sudan-perspectives of carers and psychiatrists. BMC health services research. 2015;16(1):31.

32. McDaid D, Knapp M, Raja S. Barriers in the mind: promoting an economic case for mental health in low-and middle-income countries. World Psychiatry. 2008;7(2):79-86.

33. Rugema L, Krantz G, Mogren I, Ntaganira J, Persson M. "A constant struggle to receive mental health care": health care professionals' acquired experience of barriers to mental health care services in Rwanda. BMC psychiatry. 2015;15(1):314.

34. Ssebunnya J, Kigozi F, Ndyanabangi S, Cooper S. Stakeholders' perceptions of the main challenges facing Uganda's mental health care system: a qualitative analysis. International Journal of Culture and Mental Health. 2011;4(1):54-67.

35. Strumpher J, van Rooyen D, Topper K, Andersson L, Schierenbeck I. Barriers to accessing mental health in Eastern Cape Province of South Africa. Africa Journal of Nursing and Midwifery. 2014;16(1):45-59.

36. Kpobi L, Swartz L, Ofori-Atta AL. Challenges in the use of the mental health information system in a resource-limited setting: lessons from Ghana. BMC health services research. 2018;18(1):98.

37. Sun KS, Lam TP, Lam KF, Lo TL. Barriers and facilitators for psychiatrists in managing mental health patients in Hong Kong-Impact of Chinese culture and health system. Asia-Pacific Psychiatry. 2018;10(1):e12279.

38. Wakida EK, Obua C, Rukundo GZ, Maling S, Talib ZM, Okello ES. Barriers and facilitators to the integration of mental health services into primary healthcare: a qualitative study among Ugandan primary care providers using the COM-B framework. BMC health services research. 2018;18(1):890.

39. Murphy A, Chikovani I, Uchaneishvili M, Makhashvili N, Roberts B. Barriers to mental health care utilization among internally displaced persons in the republic of Georgia: a rapid appraisal study. BMC health services research. 2018;18(1):306.

40. Wakida EK, Okello ES, Rukundo GZ, Akena D, Alele PE, Talib ZM, et al. Health system constraints in integrating mental health services into primary healthcare in rural Uganda: perspectives of primary care providers. International Journal of Mental Health Systems. 2019;13(1):16.

41. Chisholm D, Docrat S, Abdulmalik J, Alem A, Gureje O, Gurung D, et al. Mental health financing challenges, opportunities and strategies in low-and middle-income countries: findings from the Emerald project. BJPsych open. 2019;5(5). 
42. Caplan S, Little TV, Reyna P, Sosa Lovera A, Garces-King J, Queen K, et al. Mental health services in the Dominican Republic from the perspective of health care providers. Global public health. 2018;13(7):874-98.

43. Zhang Q-E, Ng CH, Ungvari GS, Van I-K, Wang G, Xiang Y-T. Mental Health-Care Delivery for Older People in China: An Update of Challenges and Recent Clinical Research. SAGE Publications Sage CA: Los Angeles, CA; 2019.

44. Jack-Ide IO, Uys L. Barriers to mental health services utilization in the Niger Delta region of Nigeria: service users' perspectives. The Pan African Medical Journal. 2013;14.

45. Hailemariam M, Fekadu A, Prince M, Hanlon C. Engaging and staying engaged: a phenomenological study of barriers to equitable access to mental healthcare for people with severe mental disorders in a rural African setting. International journal for equity in health. 2017;16(1):156.

46. Iseselo MK, Ambikile JS. Medication challenges for patients with severe mental illness: experience and views of patients, caregivers and mental health care workers in Dar es Salaam, Tanzania. International journal of mental health systems. 2017;11(1):17.

47. Nickels SV, Campos Tomasino M, Arvaiza F, Nelson A, Hunter CA. Access to mental health care in El Salvador: a case study of progress toward decentralization. Revista Panamericana de Salud Pública. 2018;42:e172.

48. Husain W. Barriers in Seeking Psychological Help: Public Perception in Pakistan. Community mental health journal. 2019:1-4.

49. James BO, Thomas FI, Seb-Akahomen OJ, Igbinomwanhia NG, Inogbo CF, Thornicroft G. Barriers to care among people with schizophrenia attending a tertiary psychiatric hospital in Nigeria. South African Journal of Psychiatry. 2019;25(1):1-6.

50. Baldisserotto ML, Theme MM, Gomez LY, Dos Reis TBQ. Barriers to seeking and accepting treatment for perinatal depression: a qualitative study in Rio de Janeiro, Brazil. Community mental health journal. 2019:1-8.

51. Rad EH, Amirbeik L, Hajizadeh M, Yousefzadeh-Chabok S, Mohtasham-Amiri Z, Rezaei S, et al. Determinants of utilization and out-of-pocket payments for psychiatric healthcare in Iran. Journal of Public Mental Health. 2019.

52. Tirintica AR, Andjelkovic I, Sota O, Pirlog MC, Stoyanova M, Mihai A, et al. Factors that influence access to mental health services in South-Eastern Europe. International journal of mental health systems. 2018;12(1):75.

53. Yu S, Kowitt SD, Fisher EB, Li G. Mental health in China: stigma, family obligations, and the potential of peer support. Community mental health journal. 2018;54(6):757-64.

54. Tristiana RD, Yusuf A, Fitryasari R, Wahyuni SD, Nihayati HE. Perceived barriers on mental health services by the family of patients with mental illness. International journal of nursing sciences. 2018;5(1):63-7.

55. Critical Appraisal Skills Programme checklists [Internet]. 2019 [cited 1/11/2019]. Available from: https://casp-uk.net/casp-tools-checklists/. 
56. Von Elm E, Altman DG, Egger M, Pocock SJ, Gøtzsche PC, Vandenbroucke JP. The Strengthening the Reporting of Observational Studies in Epidemiology (STROBE) statement: guidelines for reporting observational studies. Annals of internal medicine. 2007;147(8):573-7.

57. Bengtsson M. How to plan and perform a qualitative study using content analysis. NursingPlus Open. 2016;2:8-14.

58. Thomas J, Harden A. Methods for the thematic synthesis of qualitative research in systematic reviews. BMC medical research methodology. 2008;8(1):45.

59. Erickson CD. On the stigma of mental illness: practical strategies for research and social change. Psychiatric Services. 2006;57(7):1048-.

60. Kakuma R, Minas H, Van Ginneken N, Dal Poz MR, Desiraju K, Morris JE, et al. Human resources for mental health care: current situation and strategies for action. The Lancet. 2011;378(9803):1654-63.

61. Jenkins R, Baingana F, Ahmad R, McDaid D, Atun R. Social, economic, human rights and political challenges to global mental health. Mental Health in Family Medicine. 2011;8(2):87.

62. Saxena S, Thornicroft G, Knapp M, Whiteford H. Resources for mental health: scarcity, inequity, and inefficiency. The lancet. 2007;370(9590):878-89.

63. Patel V. Recruiting doctors from poor countries: the great brain robbery? Bmj. 2003;327(7420):926-8.

64. Hanlon C, Luitel NP, Kathree T, Murhar V, Shrivasta S, Medhin G, et al. Challenges and opportunities for implementing integrated mental health care: a district level situation analysis from five low-and middle-income countries. PloS one. 2014;9(2):e88437.

65. Collins PY, Patel V, Joestl SS, March D, Insel TR, Daar AS, et al. Grand challenges in global mental health. Nature. 2011;475(7354):27.

66. Knitzer J, Cooper J. Beyond integration: Challenges for children's mental health. Health Affairs. 2006;25(3):670-9.

67. WHO. Everybody's business: strengthening health systems to improve health outcomes: WHO's framework for action. Geneva: World Health Organization; 2007.

68. Ndetei DM, Jenkins R. The implementation of mental health information systems in developing countries: Challenges and opportunities. Epidemiology and Psychiatric Sciences. 2009;18(1):12-6.

69. Penn DL, Couture SM. Strategies for reducing stigma toward persons with mental illness. World Psychiatry. 2002;1(1):20.

70. Corrigan PW, Morris SB, Michaels PJ, Rafacz JD, Rüsch N. Challenging the public stigma of mental illness: a meta-analysis of outcome studies. Psychiatric services. 2012;63(10):963-73.

71. Sharp M-L, Fear NT, Rona RJ, Wessely S, Greenberg N, Jones N, et al. Stigma as a barrier to seeking health care among military personnel with mental health problems. Epidemiologic reviews. 2015;37(1):144-62.

72. Corrigan PW, Rao D. On the self-stigma of mental illness: Stages, disclosure, and strategies for change. The Canadian Journal of Psychiatry. 2012;57(8):464-9. 
73. Mittal D, Sullivan G, Chekuri L, Allee E, Corrigan PW. Empirical studies of self-stigma reduction strategies: a critical review of the literature. Psychiatric Services. 2012;63(10):974-81.

74. Chikovani I, Makhashvili N, Gotsadze G, Patel V, McKee M, Uchaneishvili M, et al. Health service utilization for mental, behavioural and emotional problems among conflict-affected population in Georgia: a cross-sectional study. PloS one. 2015;10(4):e0122673.

75. Pierce D, Brewer C. Factors promoting use of mental health services in a rural area of Australia. J Comm Med Health Educ. 2012;2:2161-0711.1000190.

76. Cadigan JM, Lee CM, Larimer ME. Young adult mental health: A prospective examination of service utilization, perceived unmet service needs, attitudes, and barriers to service use. Prevention Science. 2019;20(3):366-76.

77. Wiley-Exley E. Evaluations of community mental health care in low-and middle-income countries: a 10-year review of the literature. Social science \& medicine. 2007;64(6):1231-41.

78. Jacob K, Sharan P, Mirza I, Garrido-Cumbrera M, Seedat S, Mari JJ, et al. Mental health systems in countries: where are we now? The Lancet. 2007;370(9592):1061-77.

79. Whittle EL, Fisher KR, Reppermund S, Lenroot R, Trollor J. Barriers and enablers to accessing mental health services for people with intellectual disability: a scoping review. Journal of Mental Health Research in Intellectual Disabilities. 2018;11(1):69-102.

80. Stroud P, Lockwood C, Abbey J. Obstacles to the take-up of mental health care provision by adult males in rural and remote areas of Australia: a systematic review. JBI Database of Systematic Reviews and Implementation Reports. 2014;12(3):166-233.

81. Mehta N, Kassam A, Leese M, Butler G, Thornicroft G. Public attitudes towards people with mental illness in England and Scotland, 1994-2003. The British Journal of Psychiatry. 2009;194(3):278-84.

\section{Tables}

\section{Table 1. Search strategy for barriers to the provision and utilization of mental health services}

PubMed, Scopus, Web of Science, Embase, ProQuest, Wiley online library, Searched and ScienceDirect

\section{\#1 AND \#2 AND \#3}

Databases

"Mental health" OR "mental health care" OR "mental health services" OR Search Strategy "mental illnesses" OR "mental disorder" OR "mental diseases" OR psychiatry OR psychology OR psychiatric

Delivery OR provision OR providing OR utilization OR use OR usage OR \#2 access OR accessibility

Challenges OR problems OR shortcomings OR barriers OR deficits OR \#3 obstacles

Language: articles with at least an abstract in English

Limitations

Time: January 2000 - November 2019

Article type: original researches and discussion papers 
Table 2. Characteristics of studies included in the synthesis

\begin{tabular}{|c|c|c|}
\hline \multirow[t]{2}{*}{ Characteristics } & \multicolumn{2}{|c|}{ Frequency and (\%) of Included Studies } \\
\hline & MH Services Provision & MH Services Utilization \\
\hline \multicolumn{3}{|l|}{ Publication year } \\
\hline $2006-2010$ & $3(15)$ & $2(8.3)$ \\
\hline 2011-2015 & $4(20)$ & $4(16.7)$ \\
\hline $2016<$ & $13(65)$ & $18(75)$ \\
\hline \multicolumn{3}{|l|}{ Region } \\
\hline Africa & $9(45)$ & $8(33.3)$ \\
\hline Asia & $4(20)$ & $7(29.2)$ \\
\hline America & $2(10)$ & $5(20.8)$ \\
\hline Europe & $2(2)$ & $4(16.7)$ \\
\hline Multi-country & $3(15)$ & - \\
\hline \multicolumn{3}{|l|}{ Participants } \\
\hline Healthcare professional & $14(70)$ & $12(50)$ \\
\hline Managers/Policy makers & $9(45)$ & $6(25)$ \\
\hline Patients & $3(15)$ & $10(41.7)$ \\
\hline Patients' family/General population & - & $10(41.7)$ \\
\hline \multicolumn{3}{|l|}{ Study design/ type } \\
\hline Qualitative & $13(65)$ & $14(58.3)$ \\
\hline Mixed method & $3(15)$ & $7(29.2)$ \\
\hline Discussion paper & $4(20)$ & - \\
\hline Observational & - & $3(12.5)$ \\
\hline
\end{tabular}

Table 3. Themes and sub-themes on barriers to the provision of mental health services 


\begin{tabular}{|c|c|c|}
\hline \multirow[t]{2}{*}{ Themes and Sub-themes } & \multicolumn{2}{|c|}{ Included Studies } \\
\hline & $\begin{array}{l}\text { Frequency } \\
\text { (\%) }\end{array}$ & References \\
\hline \multicolumn{3}{|l|}{ Resource and administrative barriers } \\
\hline Insufficient resources & $17(85)$ & $\begin{array}{l}(10-13,15,23,31-35,38- \\
43)\end{array}$ \\
\hline $\begin{array}{l}\text { Geographical imbalance in resource } \\
\text { allocation }\end{array}$ & $7(35)$ & $(10-13,31,41,43)$ \\
\hline Centralized and non-integrated services & $7(35)$ & $\begin{array}{l}(10,12,13,20,31,38 \\
40)\end{array}$ \\
\hline Inappropriate service types & $5(25)$ & $(10,13,15,40,43)$ \\
\hline Imperfect inter-organizational collaboration & $3(15)$ & $(10,11,20)$ \\
\hline Weakness of quality assurance programs & $2(10)$ & $(11,23)$ \\
\hline \multicolumn{3}{|l|}{ Information and knowledge barriers } \\
\hline Inappropriate professionals' training & $6(30)$ & $(23,32,38,40,42,43)$ \\
\hline Imperfect mental health information system & $5(25)$ & $(11,13,20,36,39)$ \\
\hline Weakness of evidence-based practice & $5(25)$ & $(10,12,13,38,43)$ \\
\hline \multicolumn{3}{|l|}{ Policy and legislation barriers } \\
\hline Low priority of mental health in health policy & $7(35)$ & $(10-13,32,34,39)$ \\
\hline Weakness of evidence-based policy making & $4(20)$ & $(11,13,32,34)$ \\
\hline Imperfect legislation on insurance & $2(10)$ & $(15,20)$ \\
\hline $\begin{array}{l}\text { Inappropriate legislation on mental health } \\
\text { services }\end{array}$ & $2(10)$ & $(34,43)$ \\
\hline
\end{tabular}

Table 4. Themes and sub-themes on barriers to the utilization of mental health services 


\begin{tabular}{|c|c|c|}
\hline \multirow[t]{2}{*}{ Themes and Sub-Themes } & \multicolumn{2}{|r|}{ Included Studies } \\
\hline & $\begin{array}{l}\text { Frequency } \\
\text { (\%) }\end{array}$ & References \\
\hline \multicolumn{3}{|l|}{ Attitudinal barriers } \\
\hline Concern about social stigma & $20(83.3)$ & $\begin{array}{l}(17,18,20,23-25,31,33,35,37,39,42,44,45, \\
48-50,52-54)\end{array}$ \\
\hline Personal stigma/Shame & $8(33.3)$ & $(18,20,44,48,50,52-54)$ \\
\hline $\begin{array}{l}\text { Concerns about the effectiveness of } \\
\text { services }\end{array}$ & $6(25)$ & $(18,23-25,48,49)$ \\
\hline $\begin{array}{l}\text { Concerns about attitude and behavior of } \\
\text { professionals }\end{array}$ & $6(25)$ & $(17,18,20,23,42,54)$ \\
\hline $\begin{array}{l}\text { Willingness to take alternative forms of } \\
\text { care }\end{array}$ & $5(20.8)$ & $(18,23,31,33,49)$ \\
\hline Cultural beliefs against treatment & $4(16.7)$ & $(23,35,48,52)$ \\
\hline Patients' self-reliance & $3(12.5)$ & $(17,18,49)$ \\
\hline Lack of confidence in professionals & $3(12.5)$ & $(17,48,50)$ \\
\hline Poor compliance with treatment & $1(4.2)$ & (37) \\
\hline \multicolumn{3}{|l|}{ Structural barriers } \\
\hline Cost of services & $17(70.8)$ & $\begin{array}{l}(13,17,18,23-25,31,33,35,39,42,44-46,49, \\
51,54)\end{array}$ \\
\hline $\begin{array}{l}\text { Location, distance and transportation } \\
\text { barriers }\end{array}$ & $9(37.5)$ & $(13,17,18,23,25,44,47,49,54)$ \\
\hline Lack of family or social support & $9(37.3)$ & $(18,33,35,45,46,48,49,52,53)$ \\
\hline Time-related barriers & $6(25)$ & $(17,18,37,42,44,49)$ \\
\hline Inappropriate and inflexible services & $5(20.8)$ & $(17,18,42,45,49)$ \\
\hline lack of adequate services & $5(20.8)$ & $(20,23,25,52,54)$ \\
\hline Limited insurance coverage & $5(20.8)$ & $(20,33,37,39,42)$ \\
\hline \multicolumn{3}{|l|}{ Knowledge barriers } \\
\hline $\begin{array}{l}\text { Lack of knowledge about mental health } \\
\text { problem }\end{array}$ & $11(45.8)$ & $(17,18,25,35,42,45,48-52)$ \\
\hline Lack of information on available services & $5(20.8)$ & $(17,18,25,44,49)$ \\
\hline \multicolumn{3}{|l|}{ Treatment-related barriers } \\
\hline Fear of treatment side effects & $6(25)$ & $(18,23,45,46,48,49)$ \\
\hline Long-term nature of treatment & $1(4.2)$ & (45) \\
\hline
\end{tabular}

\section{Figures}




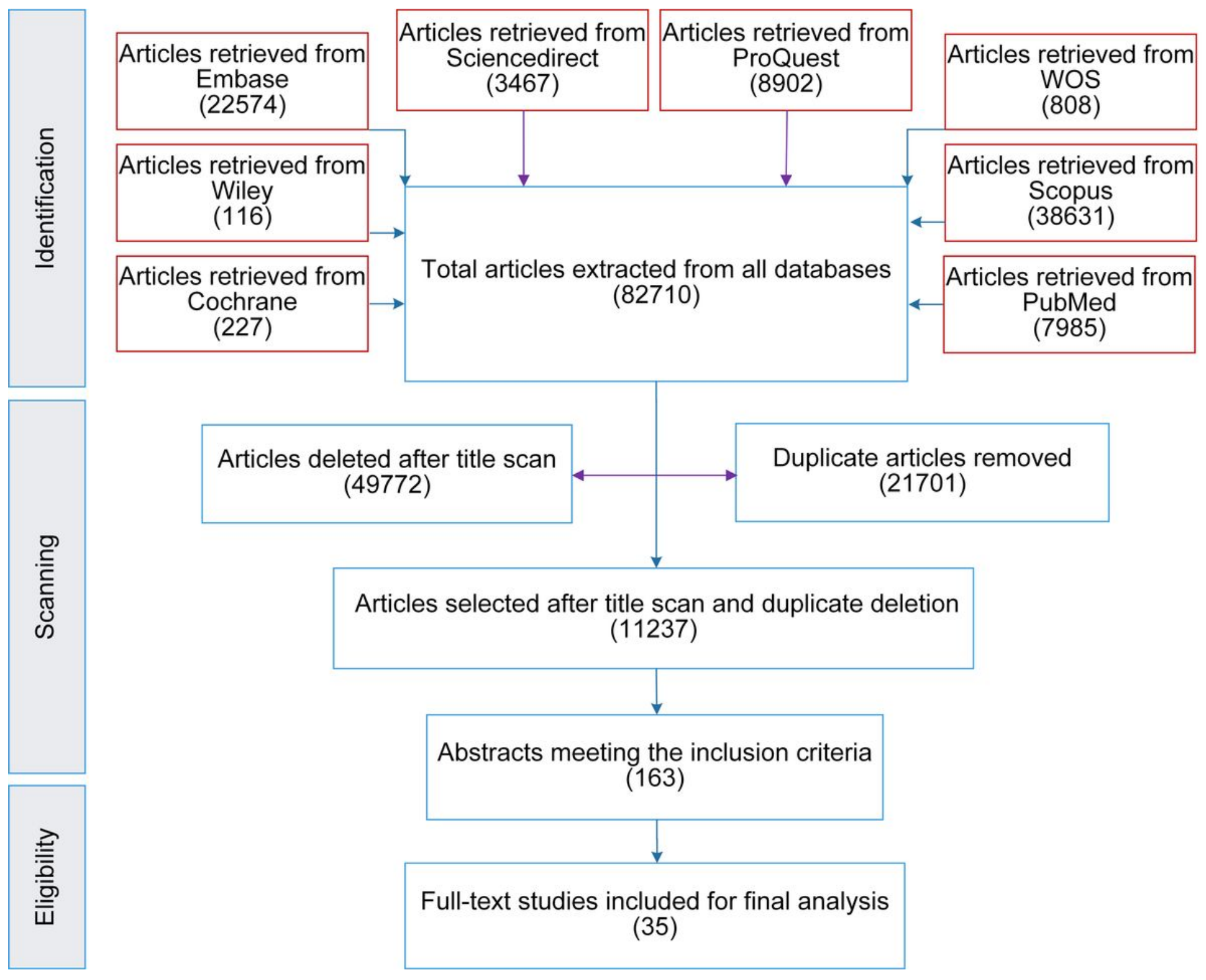

Figure 1

PRISMA Flowchart of the included papers in the scope study 


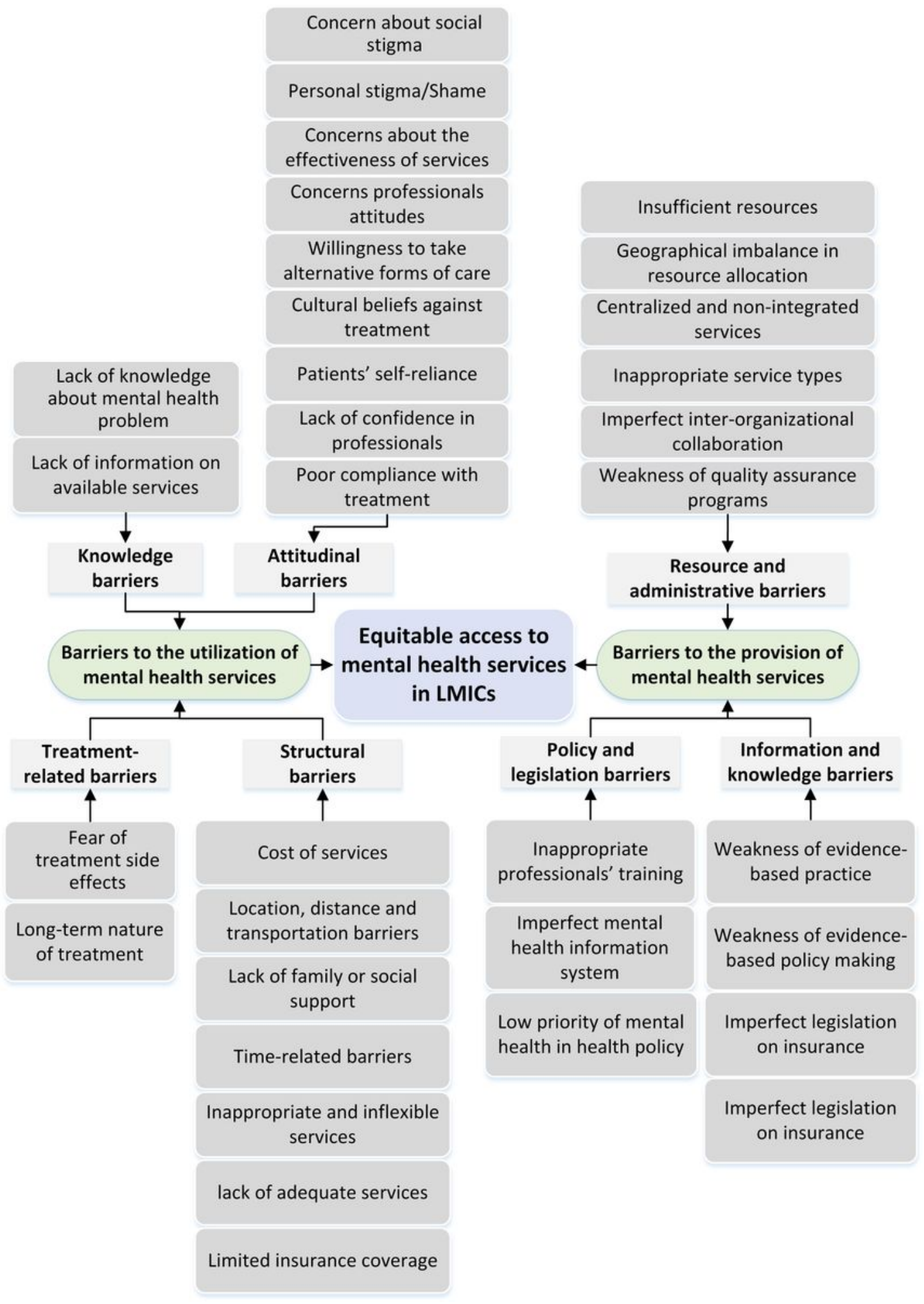

\section{Figure 2}

The thematic network of the Key Barriers to the Provision and Utilization of Mental Health Services 